\title{
LE RÔLE DES ENTREPRISES ET LEURS COMPORTEMENTS SUR LA STABILITÉ DU SYSTĖME FINANGIER
}

\author{
Farelle YANDZA IKAHAUD ${ }^{\mathrm{a}, \mathrm{b} *}$, Mohamed EL HADDAD ${ }^{\mathrm{a}}$ \\ a) University Mohammed V Agdal, Faculty of Law, Economic and Social Sciences, \\ Rabat, Maroc \\ b) Babeş-Bolyai University, Faculty of Economics and Business Administration, \\ Cluj-Napoca, Romania
}

Please cite this article as:

Yandza Ikahaud, F. and El Haddad, M., 2021. Le rôle des entreprises et leurs comportements sur la stabilité du système financier. Review of Economic Studies and Research Virgil Madgearu, 14(2), pp.85-103. doi: 10.24193/RVM.2021.14.81.
Article History:

Received: 18 October 2021 Accepted: 26 November 2021

Abstract: Investment determines the sustainability and prosperity of an economy. The paper seeks to (1) give an overview of the roles of companies on the stability of the financial system, based on existing theoretical and empirical work; and (2) to highlight the reasons which expose them to the non-application of their duties against the State. For this purpose, a bibliometric analysis using the Direct Science database was employed. The results of the analysis allowed us to study the search trend by analyzing the distribution of publications, authors, types of publications and fields of research and also showed that the themes of the study are of great scientific interest because of the surge in the number of publications on the role of companies over the last ten years averaging 76.3 per year while 1,690 were on the impact of company behaviour on the stability of the financial system.

Key words: entrepreneurship; management; finance; economy; social responsibility; business

JEL Classification: $G 1 O$; $G 32$; D8O

(C) 2021 Alma Mater Publishing House. All rights reserved.

* Corresponding author. E-mail address: farelle.yandza@econ.ubbcluj.ro. 
Review of Economic Studies and Research Virgil Madgearu, 2021, 14(2)

\section{Bibliographie:}

1. Abraham, Y.M., 2006. L’entreprise est-elle nécessaire? In: J.P. Dupuis, ed. 2007, Sociologie de l'entreprise, Montréal: Gaëtan Morin Éditeur.

2. Aloui, A., 2016. Systèmes d'Information. Polycopie de cours. [online] Available at: <https://elearning.univ-bejaia.dz/course/ view.php?id=3731> [Accessed 12 November 2021].

3. Banque de France, 2020. L'Eco en bref, La stabilité financière. Rubrique $A B C$ de l'économie. [online] Available at: <https://abceconomie.banque-france.fr/la-stabilite-financiere-o $>$ [Accessed 12 November 2021].

4. Baas, J., Schotten, M., Plume, A.M., Cote, G. and Karimi, R., 2020. Scopus as a curated, high-quality bibliometric data source for academic research in quantitative science studies. Quantitative Science Studies, 1(1), pp. 377-386. https://doi.org/10.1162/ qSs_a_ooo19.

5. Bressy, G. and Konkuyt, C., 1993. Economie d'entreprise, série aidemémoire, édition Dalloz. Paris: SIREY.

6. Bruyat, C., 2006. Création d'entreprise: Contribution Epistémologiques et Modélisation. Gestion et management, Université Pierre Mendès-France - Grenoble II, 1993, Mémoire de Thèse. [online] Available at: <https://tel.archives-ouvertes.fr> [Accessed 4 December 2021].

7. Dees, J.G., 1998. The Meaning of "Social Entrepreneurship", Working paper, [online] Available at: <https://centers.fuqua. duke.edu/case/wp-content/uploads/sites/7/2015/o3/Article_ Dees_MeaningofSocialEntrepreneurship_2001.pdf> [Accessed 4 December 2021].

8. De Moya-Anegón, F., Chinchilla-Rodríguez, Z., Vargas-Quesada, B. Corera-Álvarez, E., Muñoz-Fernández, F.J., González-Molina, A. and Herrero-Solana, V., 2007. Coverage analysis of Scopus: A journal metric approach. Scientometrics, 73 (1), pp. 53-78. https:// doi.org/10.1007/s11192-007-1681-4.

9. Drucker, P.F., 1986. Innovation and Entrepreneurship : Practice and Principles (e-book), Pan Book: business/management, Pan Books.

10. Drucker, P.F., 1974. Management: Tasks, Responsibilities, Practices (e-book), New York, NY: Harper and Row. [online] Available at: 
YANDZA IKaHAud, El HaddAd, Le Rôle DES ENTREPRises...

<https://www.academia.edu/7194379/Management_Tasks Responsibilitiesit_Peter_Drucker_> [Accessed 3 December 2021].

11. El Ghazali, M'B., Abraber, F.A. and Biyad, S., 2019. La RSE levier de création de valeur pour un entrepreneur. Revue Marocaine de la Prospective en Sciences de Gestion, [S.l.], (2), pp.1-19. Available at: <https://revues.imist.ma/index.php/RMPSG/article/ view/16625/9142> [Accessed 3 December 2021].

12. Font, A., (n.d). Théories de l'administration et politiques de gestion: Les finalités et les objectifs de l'entreprise. [online] Available at: $<$ http://andre.font.free.fr/Font/administration.html> [Accessed 10 November 2021].

13. Friedman, M., 1962. Capitalism and freedom. [online] Available at: https:// scholar.google.com/scholar_url?url=https://www.aboutthestories. com/s/Capitalism-And-Freedom.pdf\&hl=fr\&sa =X\&ei=rVirYZiyIaTy9YP 8 feA4AQ\&scisig =AAGBfm1VaryqC1XcYUighUdkmTefhkfWw\&oi=scholarr [Accessed 12 November 2021].

14. Friedman, M., 1970. The Social Responsibility Of Business Is to Increase Its Profits, The New York Times, (e-journal) [online] Available at: <https://www.nytimes.com/1970/o9/13/archives/afriedman-doctrine-the-social-responsibility-of-business-is-to. html $>$ [Accessed 3 December 2021].

15. Garrido-Cardenas, J.A., Esteban-García, B., Agüera, A., SánchezPérez, J.A. and Manzano-Agugliaro, F., 2019. Wastewater Treatment by Advanced Oxidation Process and Their Worldwide Research Trends. International Journal of Environmental Research and Public Health, 17(1), 170. https://doi.org/10.339o/ijerph17010170.

16. Gomes, P.Y., 2019. Chronique du monde. [online] Available at: $<$ https://pierre-yves-gomez.fr/le-but-de-lentreprise-nest-pas-defaire-des-profits/> [Accessed 12 November 2021].

17. Hood, W.W. and Wilson, C.S., 2001. The Literature of Bibliometrics, Scientometrics, and Informetrics. Scientometrics, 52(2), pp.291314. https://doi.org/10.1023/A:1017919924342.

18. Ionos, 2019. Startup Guide [online] Available at: <https://www. ionos.fr/startupguide/gestion/quest-ce-que-linsolvabilite/:> [Accessed 12 November 2021].

19. Jensen, M.C., 2002. Value Maximization, Stakeholder Theory, and the Corporate Objective Function. Business Ethics Quarterly, 12(2) pp.235-256. http://dx.doi.org/10.2307/3857812. 
20. Khemir, S. and Baccouche, C., 2010. Analysis of the determinants of corporate social responsibility disclosure in the annual reports of Tunisian listed firms. Research in Accounting in Emerging Economies, 10, pp.119-144. https://doi.org/10.1108/S14793563(2010)0000010010.

21. Kirzner, I.M., 1973. Competition and entrepreneurship (pdf) University of Illinois at Urbana-Champaign's Academy for Entrepreneurial Leadership Historical Research Reference in Entrepreneurship Available at: SSRN: https://ssrn.com/ abstract $=1496174$ [Accessed 1st November 2021].

22. Lasry, S. and Alaoui, F.Z., 2004. La création d'entreprise: Cas de création d'un établissement de formation professionnelle privé. Mémoire du cycle supérieur de gestion. Institut Supérieur de Commence et d'administration des entreprises (Maroc) [online] Available at: <https://wikimemoires.net/2013/04/la-creationd-entreprise-cas-un-etablissement-de-formation/> [Accessed 12 November 2021].

23. Lazreg, M., 2020. Plaidoyer pour un développement durable et une responsabilité sociale des entreprises (RSE) en Algérie. Journal des Sciences Sociales et Humaines, 13(01), pp.174-190.

24. Ouattara, L.Y., Kouassi, E.K.A., Soro, D., Soro, Y., Yao, K. B., Adouby, K., Drogui, A.P., Tyagi, D.R., and Aina, P.M., 2021. Cocoa pod husks as potential sources of renewable high-value-added products: A review of current valorizations and future prospects. BioResources, 16(1), pp.1988-2020.

25. Palmatier, R.W., Houston, M.B. and Hulland, J., 2018. Review articles: purpose, process, and structure. Journal of the Academy of Marketing Science, 46, pp. 1-5. https://doi.org/10.1007/s11747017-0563-4.

26. Perroux, F., 1965. L'économie du XXème siècle, 2ème éd. Paris: PUF.

27. Rip, A., 1997. Qualitative conditions of scientometrics: The new challenges. Scientometrics, 38 (1), pp. 7-26.https://doi.org/10.1007/ BF02461120.

28. Serenko, A., Bontis, N., Booker, L., Sadeddin, K. and Hardie, T., 2010. A scientometric analysis of knowledge management and intellectual capital academic literature (1994-2008). Journal of Knowledge Management, 14(1), pp.3-23. https://doi. org/10.1108/13673271011015534. 
29. Snyder, H., 2019. Literature review as a research methodology: An overview and guidelines. Journal of Business Research, 104(1), pp.333-339. https://doi.org/10.1016/j.jbusres.2019.07.039.

30. Tan, J., Fu, H.Z. and Ho, Y.S., 2014. Abibliometric analysis of research on proteomics in science citation index expanded. Scientometrics, 98, pp.1473-1490. https://doi.org/10.1007/s11192-013-1125-2.

31. Zupic, I. and Čater, T., 2015. Bibliometric Methods in Management and Organization. Organizational Research Methods, 18(3), pp.429472. https://doi.org/10.1177/1094428114562629.

32. ${ }^{* *}$, Wilkipedia., 2017. Entreprise [online] Available at: <https:// fr.wikipedia.org/wiki/Entreprise > [Accessed 12 November 2021]. 